\title{
The Effects of Aging on Substantia Nigra Dopamine Neurons
}

\author{
Kauê M. Costa \\ Institute for Neurophysiology, Neuroscience Center, Goethe University, 60590 Frankfurt am Main, Germany \\ Review of Branch et al.
}

Aging beyond young adulthood is associated with accumulating deficits in cognition and motor control, as well as an increased chance of developing neurodegenerative disorders. But how aging affects the physiology of vulnerable neurons and how this leads to age-related functional deficits are not fully understood. Among the neuronal populations most affected by aging, dopaminergic neurons of the substantia nigra pars compacta are of particular interest. These neurons project to the dorsal striatum where their patterns of dopamine release are essential for volitional motor control and several cognitive functions (Paladini and Roeper, 2014). Previous studies have shown that nigral dopamine neurons are progressively lost during normal aging (Gibb and Lees, 1991), which likely contributes to age-related functional deficits. Moreover, old age is the major risk factor for Parkinson's disease, which is characterized by a tenfold accelerated loss of nigral dopamine neurons (Gibb and Lees, 1991). A key question is whether functional changes in surviving old nigral dopamine neurons also contribute to the aging phenotype.

In a recent study published in The Journal of Neuroscience, Branch and col-

Received Sept. 8, 2014; revised 0ct. 8, 2014; accepted 0ct. 10, 2014.

K.M.C. is a member of the doctoral program of the International Max Planck Research School for Neural Circuits (Max Planck Institute for Brain Research, Germany). The author thanks Jochen Roeper for his helpful comments and suggestions.

The author declares no competing financial interests.

Correspondence should be addressed to Kauê Machado Costa, Institute of Neurophysiology, Haus 89, Goethe University Frankfurt, Theodor-Stern-Kai 7 60590, Frankfurt am Main, Germany. E-mail: kaue.m.costa@gmail.com.

DOI:10.1523/JNEUROSCI.3739-14.2014

Copyright $\odot 2014$ the authors $\quad 0270-6474 / 14 / 3415133-02 \$ 15.00 / 0$ leagues (2014) made a substantial contribution by investigating how age affects several electrophysiological properties of nigral dopamine neurons in vitro. Their first feat was obtaining viable midbrain slices from mice that were at the limits of their life expectancy (25-30 months). In such preparations, dopamine neurons exhibit slow (1-8 Hz) spontaneous pacemaker firing generated by intrinsic ionic currents. Using extracellular cell-attached recordings, Branch and colleagues (2014) discovered that nigral dopamine neurons from old mice had significantly lower spontaneous firing rates, shorter spike widths, and lower pacemaker precision (i.e., higher variability in interspike interval) than neurons from young adult (2-7 months) counterparts. Whole-cell patchclamp recordings revealed that the input resistance and capacitance of old and young neurons were similar, indicating that the observed slower firing was unrelated to changes in resting membrane conductances or gross morphology.

The authors next used biophysical and pharmacological approaches to identify the relevant changes in ion channels that caused the age-related change in firing. Surprisingly, they found that several key channels that control dopamine neuron pacemaker rate and precision, including hyperpolarizationactivated cyclic nucleotide-gated (HCN) channels and small conductance calciumactivated potassium (SK) channels (Paladini and Roeper, 2014), were unaffected by aging. These "negative" findings are important, because they show that aging does not affect all ion channels involved in pacemaker firing.
The authors identified only one membrane current that was altered by age: the L-type calcium current, which in dopamine neurons is mediated by $\mathrm{Ca}_{\mathrm{V}} 1.3$ and $\mathrm{Ca}_{\mathrm{V}} 1.2 \alpha$ subunits. The effect of age on L-type currents was substantial, with the average L-type current amplitude being nearly threefold smaller in old neurons than in young neurons. Moreover, blocking this current with nimodipine lowered firing rate and precision in both young and old neurons, suggesting that the reduction of L-type currents likely contributes to the age-related effects on pacemaker firing.

The precise role of L-type channels for pacemaker firing in adult nigral dopamine neurons is still disputed. Some of these neurons exhibit a sinusoidal subthreshold voltage oscillation that depends on functional voltage-gated calcium channels. Some studies argue that pacemaker firing is generated by this oscillation, and that blocking L-type channels disrupts pacemaker firing by removing a crucial driving force for the depolarizing phase of the oscillation (Putzier et al., 2009a). Others, however, argue that subthreshold oscillations are not essential for spontaneous firing and that blocking L-type channels does not affect pacemaker firing itself(Guzman et al., 2009). One explanation for these conflicting findings is that nigral dopamine neurons are not homogeneous with respect to L-type channel function (Roeper, 2013). In any case, in Branch and colleagues' (2014) study L-type channels appear to play a key role in controlling pacemaker firing.

Interestingly, the authors found that nimodipine had an unexpected biphasic effect on firing rate in young neurons, first increasing then decreasing spike fre- 
quency. In old neurons, there was only a monophasic slowing of firing, which is expected in response to inhibiting a depolarizing current. This feature cannot be explained exclusively by the age-related reduction in L-type current, which would not account for the firing rate increase in young neurons. One possibility is that aging might affect the functional coupling of L-type channels to intracellular calcium stores and in turn to calcium-activated potassium channels. Therefore, the biphasic frequency effect of nimodipine in young neurons might have been caused by an indirect effect of L-type channel inhibition on calciumdependent hyperpolarizing mechanisms, which might be blunted in old neurons.

While future studies will need to confirm or reject this hypothesis, the finding suggests that other, as yet unknown, mechanisms may contribute to age-related changes in pacemaker control. Unfortunately, Branch and colleagues (2014) did not state whether nimodipine abolished the differences in spike width between old and young neurons, which would have both causally implicated the L-type current reduction in age-related spike shortening and help rule out changes in other channels that determine spike waveform, such as voltage-gated potassium channels.

The authors also showed, using reverse transcription real-time quantitative PCR of dissected nigral tissue, that expression levels of mRNA encoding $\mathrm{Ca}_{\mathrm{v}} 1.2$ and $\mathrm{Ca}_{\mathrm{v}} 1.3 \alpha$ subunits were similar in aged and young mice. However, this does not rule out the involvement of more subtle transcriptional mechanisms for reduced L-type currents, such as changes in expression of auxiliary subunits, or possible changes in the expression of specific $\alpha$ subunit splice variants. Nevertheless, the data indicates that age-related changes in L-type channels might be caused by a yet unknown posttranslational mechanism, such as altered channel phosphorylation. Clearly more studies are needed to determine the full spectrum of dopamine neuron channels affected by age, as well as the molecular underpinnings of these changes.

In addition to mechanistic issues, the study by Branch and colleagues (2014) raises another important question: how does the age-related decrease in L-type currents affect the in vivo firing pattern of dopamine neurons and dopaminedependent behavior? One important facet of this question is the possible effect on in vivo burst firing. In vitro, dopamine neurons fire only as pacemakers, but in vivo these neurons also show spontaneous and context-driven bursts (transient increases in firing rate $>10 \mathrm{~Hz}$ ) that are triggered by glutamatergic synaptic input and are essential for phasic striatal dopamine release (Paladini and Roeper, 2014). Dynamicclamp studies have shown that L-type channels might amplify the maximum firing rate of NMDAR-generated bursts (Deister et al., 2009). If this applies to in vivo bursts - which remains to be experimentally demonstrated-an age-related reduction in L-type currents might result in reduced intraburst frequencies and possibly lower the efficacy with which NMDAR activation generate bursts. This, in turn, could limit the amplitude of phasic striatal dopamine signals, as well as distort their temporal locking to sensory and motor signals. Such a potential compression in dynamic range of dopamine signaling might itself be a candidate mechanism for agerelated motor and cognitive deficits. It could also explain why some aging deficits are ameliorated by L-DOPA, which is believed to boost mainly phasic dopamine signaling (Chowdhury et al., 2013).

However, the effects of age on pacemaker firing itself could also lead to a different in vivo phenotype. Previous studies suggest that intrinsic pacemaker irregularity in nigral dopamine neurons is positively correlated with burstiness after T-type and SK channel blockade in vitro (Wolfart and Roeper, 2002), indicating that increasing interspike interval variability facilitates bursting. In addition, slowing the spontaneous firing rate of dopamine neurons increases their response to synaptic inputs in an exponential manner, which could also promote bursting (Putzier et al., 2009b). These previous findings open the possibility that the slower and irregular tonic firing observed in old nigral dopamine neurons might potentiate burst firing in vivo. In this scenario, the observed changes in spontaneous firing could be a compensatory mechanism for maintaining phasic striatal dopamine release despite other age-related deficits in the nigrostriatal pathway, such as dopamine cell loss.

Future studies, using in vivo recordings of nigral dopamine neurons and striatal dopamine release, combined with local and ideally subtype-specific pharmacological blockade or genetic manipulation of L-type channels, will be necessary to define the in vivo consequences of age-related changes in L-type function and pacemaker activity.

Another important question is whether different subpopulations of dopamine neurons respond differently to age. For example, a subset of dorsomedial nigral dopamine neurons express calbindin-D28k and have higher survival rates in Parkinson's disease (Yamada et al., 1990). Branch and colleagues (2014) suggested that smaller L-type currents might help the survival of old dopamine neurons by decreasing calcium entry and preventing degeneration caused by disrupted calcium homeostasis, which has been proposed as a common final pathway in aging and in several neurodegenerative diseases. Considering that calbindin-D28k increases calcium buffering capacity, it would be interesting to determine whether calbindin-expressing dopamine neurons are spared from the age-related decrease in L-type current. Additionally, it would be important to differentiate how aging affects dopamine neurons of the neighboring ventral tegmental area, which also express L-type channels. In summary, the study by Branch and colleagues (2014) opens important new avenues to unravel the role of ion channels in neuronal aging.

\section{References}

Branch SY, Sharma R, Beckstead MJ (2014) Aging decreases L-type calcium channel currents and pacemaker firing fidelity in substantia nigra dopamine neurons. J Neurosci 34:9310 9318. CrossRef Medline

Chowdhury R, Guitart-Masip M, Lambert C, Dayan P, Huys Q, Düzel E, Dolan RJ (2013) Dopamine restores reward prediction errors in old age. Nat Neurosci 16:648-653. CrossRef Medline

Deister CA, Teagarden MA, Wilson CJ, Paladini CA (2009) An intrinsic neuronal oscillator underlies dopaminergic neuron bursting. J Neurosci 29: 15888-15897. CrossRef Medline

Gibb WR, Lees AJ (1991) Anatomy, pigmentation, ventral and dorsal subpopulations of the substantia nigra, and differential cell death in Parkinson's disease. J Neurol Neurosurg Psychiatry 54:388-396. CrossRef Medline

Guzman JN, Sánchez-Padilla J, Chan CS, Surmeier DJ (2009) Robust pacemaking in substantia nigra dopaminergic neurons. J Neurosci 29:1101111019. CrossRef Medline

Paladini CA, Roeper J (2014) Generating bursts (and pauses) in the dopamine midbrain neurons. Neuroscience 282C:109-121. CrossRef Medline

Putzier I, Kullmann PH, Horn JP, Levitan ES (2009a) Cav1.3 channel voltage dependence, not $\mathrm{Ca} 2+$ selectivity, drives pacemaker activity and amplifies bursts in nigral dopamine neurons. J Neurosci 29: 15414-15419. CrossRef Medline

Putzier I, Kullmann PH, Horn JP, Levitan ES (2009b) Dopamine neuron responses depend exponentially on pacemaker interval. J Neurophysiol 101: 926-933. CrossRef Medline

Roeper J (2013) Dissecting the diversity of midbrain dopamine neurons. Trends Neurosci 36: 336-342. CrossRef Medline

Wolfart J, Roeper J (2002) Selective coupling of T-type calcium channels to SK potassium channels prevents intrinsic bursting in dopaminergic midbrain neurons. J Neurosci 22: 3404-3413. Medline

Yamada T, McGeer PL, Baimbridge KG, McGeer EG (1990) Relative sparing in Parkinson's disease of substantia nigra dopamine neurons containing calbindin-D28K. Brain Res 526: 303-307. CrossRef Medline 